\title{
Wherefore art thou, chaperone?
}

\author{
Sanjeewa Sumathipala* \\ Member of the Royal College of General Practitioners, London, UK \\ Received: 25 December 2020 \\ Revised: 10 January 2021 \\ Accepted: 11 January 2021

\section{*Correspondence:} \\ Dr. Sanjeewa Sumathipala, \\ E-mail: sansumathipala@gmail.com \\ Copyright: ( ) the author(s), publisher and licensee Medip Academy. This is an open-access article distributed under \\ the terms of the Creative Commons Attribution Non-Commercial License, which permits unrestricted non-commercial \\ use, distribution, and reproduction in any medium, provided the original work is properly cited.
}

\begin{abstract}
Respect for the dignity of a patient and their privacy are fundamental concerns for all doctors. When an intimate examination is to be undertaken, that respect needs to be especially maintained. The offer of an appropriate chaperone during that examination can help to demonstrate that respect and can safeguard interests of the patient and the doctor.
\end{abstract}

Keywords: Intimate examination, Chaperone, Doctor-patient relationship

\section{INTRODUCTION}

The United Kingdom's medical regulator, general medical council (GMC) states that patients must be treated as individuals and their dignity and privacy respected. ${ }^{1}$ A patient's dignity and privacy are especially important to respected when intimate examinations are undertaken.

According to the GMC, an intimate examination is likely to include examinations of the breasts, genitalia and rectum, but may also include any examination where it is necessary to be close or even touch the patient. ${ }^{2}$

The GMC states that a patient should be offered a chaperone if an intimate examination is required. The issue of chaperones gained prominence in the UK following the Ayling inquiry in $2004 .{ }^{3}$ Dr. Ayling was convicted of 13 counts of indecent assault of patients between 1991 and 1998.

A chaperone acts as an impartial observer, safeguarding all parties (patient and doctors) and is also a witness to continuing consent of the procedure. ${ }^{2,4} \mathrm{~A}$ relative or friend of the patient is not an impartial observer and so would not usually be a suitable chaperone, but the doctor should comply with a reasonable request to have such a person present as well as a chaperone.

\section{THE DOCTOR-PATIENT RELATIONSHIP}

Trust and respect for the patient's autonomy underpins the doctor-patient relationship. ${ }^{5}$ This is especially important in primary care where a patient is likely to present to a doctor with acute or chronic conditions as the first stage of access to a healthcare system.

However, within the doctor patient relationship there is a power differential and the potential for a paternalistic, doctor knows best, relationship to develop. ${ }^{6}$ The patient is thereby vulnerable to accepting advice, tests or treatment without feeling able to disagree with a doctor. ${ }^{5}$

The globalization of medical ethics also requires the doctors to be able to appreciate the different sensitivities for the patients that they treat whilst operating within the framework of particular health care systems. Hence, in countries like Qatar, certain premedical courses have provided the aspiring doctors of the future with an understanding of a variety ethical systems. ${ }^{7}$

Appreciating cultural and religious factors provide important determinants in the doctor-patient relationship, 
in order to ensure that the patient is not unintentionally embarrassed or made to feel uncomfortable. ${ }^{8}$

\section{PREVIOUS RESEARCH ON CHAPERONES}

The question of whether a chaperone should be present during medical examinations has been debated for decades. The early belief appeared to be that the answer rested upon the doctor's discretion. A postal questionnaire was sent to 200 male UK general practitioners to assess attitudes towards chaperones and the extent of their use when female patients needed to be examined. ${ }^{9}$ Of 171 respondents, $13 \%$ claimed they always used a chaperone and $25 \%$ said they never did. Many of the general practitioners relied on 'instinct' to decide whether to use a chaperone. An equal number considered a chaperone to be detrimental to the doctorpatient relationship as said they believed the chaperone's presence to be beneficial.

Twenty years later, another questionnaire study focused on 400 primary care patients whose doctors did not routinely offer chaperones. ${ }^{10} 15 \%$ of patients always wanted a chaperone to be present-the same proportion as those opposed having a chaperone present (15\%). Women were more likely than men to think that that they should be asked if they would like a chaperone present for an intimate examination $(\mathrm{p}=0.001) .90 \%$ of females preferred a nurse to act as the chaperone, compared with only $39 \%$ male responders $(p<0.001)$. No participant considered receptionists as appropriate to act as chaperones.

Absence of consistent use of chaperones in intimate examinations is also found in studies of doctors working in secondary care. A questionnaire-based study of UK based urologists demonstrated that $72.5 \%$ always used a chaperone whilst $22.9 \%$ never used a chaperone when the patient was of the same sex. ${ }^{11} 42.5 \%$ did not feel that using a chaperone assisted the doctor's examination, and $17.2 \%$ respondents felt that chaperones were unnecessary. Of concern, $38.9 \%$ were not aware of the GMC guidance on chaperones.

The relative under usage of chaperones is reflected in several international studies. In a Hong Kong based study of 150 patients at a public teaching hospital, over $90 \%$ considered the presence of a chaperone appropriate during intimate examination, and $84 \%$ felt that doctors, whether male or female, should always request the presence of a chaperone. ${ }^{12}$ The most cited reasons included the availability of an objective account should any legal issue arise, protection against sexual harassment, and to provide psychological support.

$75 \%$ of female participants preferred a chaperone is a male doctor as undertaking an intimate physical examination and $28.6 \%$ still preferred to be chaperoned when being examined by a female doctor. Among male participants, over $50 \%$ indicated no specific preference for being chaperoned, but $21.2 \%$ preferred a chaperone to be present if a male doctor and $25.8 \%$ if a female doctor was examining them.

A questionnaire-based study of 500 Ontario members of the college of family physicians of Canada regarding intimate examinations revealed that chaperones were more commonly used with female patients than with males $(\mathrm{p}<0.001)$, with the female pelvic exam being the most likely of the five exams to be attended by a chaperone. ${ }^{13}$ With respect to the identity of the chaperone, approximately two-thirds of respondents reported using nurses as chaperones and $10 \%$ relied on the presence of a family member.

The usage of a family member, though not recommended by the GMC as an appropriate chaperone, can have more frequent usage in other societies. In a study set in the outpatient clinics of a tertiary care hospital in Riyadh, Saudi Arabia, family members comprised most of the chaperones. ${ }^{14}$ With respect to the views of physicians, $44.1 \%$ (69.2\% female vs $39 \%$ male physicians, $\mathrm{p}=0.001)$ reported that they used chaperones infrequently $(\leq 25 \%$ of the time). $58.6 \%$ of physicians in training used chaperones in comparison to $36.8 \%$ of physicians who has completed training $(p=0.007) .{ }^{15}$ Of those physicians who offered chaperones infrequently, $52.1 \%$ had undertaken post graduate training in Islamic countries whereas $35.6 \%$ had trained in in western countries, $\mathrm{p}=0.027)$.

A self-administered, questionnaire-based survey conducted in South Africa, of doctors who undertook gynecology consultations and examinations, of those who felt that it was always necessary to have a chaperone did so because of medico legal considerations. ${ }^{16}$ Of the males who felt it unnecessary to have a chaperone present, they gave no clear reasons why. Female respondents who did not feel a chaperone necessary, said that they did not consider the situation to be threatening to themselves or the patients. Moreover, the study's findings suggested that whether to use a chaperone was decided on a caseby-case basis.

Studies, across several countries demonstrate a chaperone is declined more often if offered by a female clinician compared to a male clinician. However, a proportion of female patients will still choose a chaperone even with a female clinician. ${ }^{17}$ There is an ongoing change in the gender composition of doctors across the world. ${ }^{18}$ Female medical students are increasing in relative numbers and constitute $40-60 \%$ of medical students. ${ }^{19}$ Hence, male patients may find that they are not being examined by a male doctor as often as in the past, and the request for a chaperone may become more frequent.

Studies have also shown that many patients see the offer of a chaperone as a demonstration of respect from their doctor, helping to build a good patient-doctor relationship. ${ }^{20}$ The culture of medicine decision making is 
changing with the focus should be on each individual patient decision and not on clinician presumption of preference for chaperone. ${ }^{17}$ The sensitivity of the patient, affected by a range of influences such as cultural and religious guidance, must not be overlooked by the doctor, and the value of family members present at an examination should not be discounted, but factored into how an intimate examination can proceed where neither the patient or doctor feels embarrassed or vulnerable.

\section{CONCLUSION}

The appropriate use of chaperones will help to safeguard patients and doctors. The doctor must not assume to know whether a patient would or would not prefer to have a chaperone present at an intimate examination.

There is evidence that patients interpret the offer of a chaperone as a sign of respect for the patient by the doctor. Although family members and friends are not impartial, they may provide reassurance for the patient, but the doctor should not object to their presence. Appropriate documentation of the offer of the chaperone, the patient's response, and the identity of the chaperone if accepted, is required in all cases.

An unchaperoned intimate examination is potentially fraught with danger for both the doctor and the patient and is ordinarily inadvisable. Deferring the examination until such time that a suitable chaperone can be present is preferable but ensuring that the health of the patient does not suffer in the interval.

Funding: No funding sources

Conflict of interest: None declared

Ethical approval: Not required

\section{REFERENCES}

1. Good Medical Practice. General Medical Council. 2013. Available at: https://www.gmc-uk.org//media/documents/good-medical-practice---english20200128_pdf-51527435.pdf?la=en\&hash= DA1263358CCA88F298785FE2BD7610EB4EE9A5 30. Accessed on 10 December 2020.

2. Intimate examinations and chaperones. General Medical Council. 2013. Available at: https://www.gmc-uk.org/-/media/documents/ maintaining-boundaries-intimate-examinations-andchaperones_pdf-58835231.pdf?la=en\&hash= A6DCCA363F989E0304D17FBC4ECB9C10600283 85. Accessed on 10 December 2020.

3. Department of Health. Publications, Policy and Guidance. Committee of Inquiry independent investigation into how the NHS handled allegations about the conduct of Clifford Ayling Section 2.58. Available at: http://www.dh.gov.uk/en/Publication sandstatistics/Publications/PublicationsPolicyAndGu idance/DH_408899. Accessed on 14 December 2020 .
4. Guidance on the Role and Effective Use of Chaperones in Primary and Community Care settings. MODEL CHAPERONE FRAMEWORK. NHS Clinical Governance Support Team. Available at: https://www.lmc.org.uk/visageimages/guidance/ 2007/Chaperone_model\%20framework.pdf. Accessed on 12 December 2020.

5. Van Hecke O, Jones KM. The Attitudes and Practices of General Practitioners about the Use of Chaperones in Melbourne, Australia. Int J Family Med. 2012;2012:768461.

6. Rodriguez-Osorio CA, Dominguez-Cherit G. Medical decision-making paternalism versus patientcentered (autonomous) care. Curr Opin Crit Care. 2008;14(6):708-13.

7. Del Pozo PR, Fins JJ. The Globalization of Education in Medical Ethics and Humanities: Evolving Pedagogy at Weill Cornell Medical College in Qatar. Academic Med. 2005;80(2):135-40.

8. Abdulghani HM, Haque S, Irshad M, Al-Zahrani N, Al-Bedaie E, Al-Fahad L et al. Students' perception and experience of intimate area examination and sexual history taking during undergraduate clinical skills training: A study from two Saudi medical colleges. Medicine (Baltimore). 2016;95(30):e4400.

9. Jones RH. The use of chaperones by general practitioners. J R Coll Gen Pract. 1983;33(246):25-7.

10. Whitford DL, Karim M, Thompson G. Attitudes of patients towards the use of chaperones in primary care. Br J Gen Pract. 2001;51(466):381-3.

11. Modgil V, Barratt R, Summerton DJ, Muneer A. Chaperone use amongst UK urological surgeons - an evaluation of current practice and opinion. Ann R Coll Surg Engl. 2016;98(4):268-9.

12. Fan VC, Choy HT, Kwok GY, Lam HG, Lim QY, Man YY, et al. Chaperones and intimate physical examinations: what do male and female patients want? Hong Kong Med J. 2017;23(1):35-40.

13. Price DH, Tracy CS, Upshur RE. Chaperone use during intimate examinations in primary care: postal survey of family physicians. BMC Fam Pract. 2005;6:52.

14. Al Gaai E, Al Sayed H, Hammami MM. Medical chaperoning at a tertiary care hospital in Saudi Arabia: prevalence and patient preference. Ann Saudi Med. 2007;27(3):217-9.

15. Al-Gaai EA, Hammami MM. Medical chaperoning at a tertiary care hospital in Saudi Arabia: survey of physicians. J Med Ethics. 2009;35(12):729-32.

16. Guidozzi Y, Gardner J, Dhai A. Professionalism in the intimate examination: How healthcare practitioners feel about having chaperones present during an intimate consultation and examination. S Afri Med J. 2013;103(1):25-7.

17. Rose K, Eshelby S, Thiruchelvam P. The importance of a medical chaperone: a quality improvement study exploring the use of a note stamp in a tertiary breast surgery unit. BMJ Open. 2015;5:e007319. 
18. Jefferson L, Bloor K, Maynard A. Women in medicine: historical perspectives and recent trends. Bri Med Bull. 2015;114(1):5-15,

19. Sabet F, Zoghoul S, Alahmad M, Al Qudah H. The influence of gender on clinical examination skills of medical students in Jordan: a cross-sectional study. BMC Medi Edu. 2020;20:98.
20. Sinha S, De A, Jones N, Jones M, Williams RJ, Vaughan-Williams E. Patients' attitude towards the use of a chaperone in breast examination. Ann R Coll Surg Engl. 2009; 91:46-9

Cite this article as: Sumathipala $\mathrm{S}$. Wherefore art thou, chaperone? Int J Res Med Sci 2021;9:641-4. 